\title{
Effect of Relaxin Expression from an Alginate Gel-Encapsulated Adenovirus on Scar Remodeling in a Pig Model
}

\author{
In Sik Yun ${ }^{1}$, Eunhye Kang ${ }^{1}$, Hyo Min Ahn², Yong Oock Kim ${ }^{1}$, Dong Kyun Rah ${ }^{1}$, \\ Tai Suk Roh ${ }^{1}$, Won Jai Lee ${ }^{1}$, and Chae-Ok Yun ${ }^{2,3,4}$ \\ ${ }^{1}$ Institute for Human Tissue Restoration, Department of Plastic \& Reconstructive Surgery, Yonsei University College of Medicine, Seoul; \\ ${ }^{2}$ Department of Bioengineering, College of Engineering, Hanyang University, Seoul; \\ ${ }^{3}$ Institute of Nano Science and Technology (INST), Hanyang University, Seoul; \\ ${ }^{4}$ GeneMedicine Co., Ltd., Seoul, Korea.
}

\begin{abstract}
Purpose: Relaxin (RLX) is a transforming growth factor- $\beta 1$ (TGF- $\beta 1$ ) antagonist that is believed to function as a potent collagen re-arranger and a major suppressor of extracellular matrix components. Adenoviruses (Ads) are accepted vectors for cancer gene therapy. However, repeated treatments of Ad are limited by short-term biological activity in vivo. The efficacy of sustained RLX expression to scar remodeling was assessed using an injectable alginate gel-matrix system.

Materials and Methods: Pig scar tissue was treated with relaxin-expressing Ad loaded in alginate gel (gel/Ad-RLX). Surface areas, color, and pliability of scars were compared, and various factors influencing scar formation and collagen arrangement were analyzed.

Results: Gel/Ad-RLX decreased scar size, color index, and pliability. Immunohistochemistry showed decreased levels of major extracellular matrix proteins in the gel/Ad-RLX-treated group. Furthermore, treatment with gel/Ad-RLX reduced expression of tissue inhibitor of metalloproteinase-1 and alpha-smooth muscle actin and markedly increased expression of matrix metalloproteinase-1 in pig scar tissues. Gel/Ad-RLX also significantly downregulated TGF- $\beta 1$ and upregulated TGF- $\beta 3$ mRNAs in pig scar tissues. Conclusion: These results support a prominent role for RLX in scar remodeling and suggest that gel/Ad-RLX may have therapeutic effects on scar formation.
\end{abstract}

Key Words: Gene therapy, scar remodeling, relaxin, adenovirus, alginate gel, pig scar model

Received: November 22, 2018 Revised: June 25, 2019

Accepted: July 23, 2019

Co-corresponding authors: Chae- $\mathrm{Ok}$ Yun, PhD, Department of Bioengineering, College of Engineering, Hanyang University, 222 Wangsimni-ro, Seongdong-gu, Seoul 04763, Korea.

Tel: 82-2-2220-0491, Fax: 82-2-2220-4850, E-mail: chaeok@hanyang.ac.kr and Won Jai Lee, MD, PhD, Institute for Human Tissue Restoration, Department of Plastic \& Reconstructive Surgery, Yonsei University College of Medicine, 50-1 Yonsei-ro, Seodaemun-gu, Seoul 03722, Korea.

Tel: 82-2-2228-2210, Fax: 82-2-393-6947,E-mail: pswjlee@yuhs.ac

-The authors have no potential conflicts of interest to disclose.

(C) Copyright: Yonsei University College of Medicine 2019

This is an Open Access article distributed under the terms of the Creative Commons Attribution Non-Commercial License (https://creativecommons.org/licenses/ by-nc/4.0) which permits unrestricted non-commercial use, distribution, and reproduction in any medium, provided the original work is properly cited.

\section{INTRODUCTION}

The skin is the largest organ in mammals. Skin has many important roles, including protection, maintenance of homeostasis, thermoregulation, vitamin D synthesis, and sensory perception. Damage to the skin by trauma or surgery activates a series of interdependent and sequential physiological events involving inflammation, cell proliferation, matrix deposition, and remodeling for tissue repair. Excessive collagen production and deposition during wound healing cause abnormal dermal proliferation and the formation of hypertrophic scars. Hypertrophic scars often present with redness and itching as firm, rubbery lesions or shiny, fibrous nodules on the surface of the skin. ${ }^{1,2}$

Various mechanisms have been proposed to explain the 
pathogenesis of scars. ${ }^{3}$ However, treatment is difficult due in part to the lack of an accurate, practical, reproducible, and economical animal model with which to systematically study scar remodeling. Animal models are important for studying the complex interactions that occur in living tissue during wound healing without the limitations of in vitro techniques. Currently, many researchers use rodent models to study wound healing due to low costs, small animal size, and relative ease of handling and care. However, hypertrophic scars do not develop in these species. In addition, the dermal composition of rodents does not resemble that of humans. In contrast, porcine skin is a good model for studying human dermal repair due to a number of similarities with human skin. Porcine and human skin has remarkably similar anatomies and physiologies, similar thicknesses of dermal and epidermal layers, and the presence of similar dermal appendages ${ }^{4-7}$ In addition, juvenile domestic pigs can be used instead of adult swine to address difficult handling and housing issues. Although juvenile animals tend to heal quicker than their older counterparts, the healing process takes a few months in juvenile pigs. Thus, sufficient time is available to assess the physiology and cellular biology of the healing process in a juvenile porcine model. ${ }^{8}$

The 6-kDa polypeptide hormone relaxin (RLX) is a member of the insulin family of peptides. RLX is primarily produced by the ovaries and/or placenta during pregnancy in mammals. RLX has multiple pregnancy-related functions, including connective tissue remodeling in the reproductive tract and cervix during pregnancy and softening of the cervix and vagina at the time of delivery. ${ }^{9}$ Recent studies have demonstrated that RLX also has biological effects on the brain, heart, kidneys, lungs, and connective tissues independent of its roles in reproduction. ${ }^{10-12}$ These findings suggest that RLX may have therapeutic applications for non-reproductive conditions, such as fibrosis. RLX may reduce fibrosis by suppressing collagen synthesis and increasing collagenase activity with subsequent collagen degradation. . $^{13-15}$

During the past 20 years, the field of virus-mediated gene therapy has grown tremendously. Adenoviruses (Ads)-based vectors, in particular, have attracted attention as gene-delivery vehicles due to their high titer capability and transduction efficiency in both dividing and non-dividing cells. ${ }^{16,17}$ In this study, we introduced a RLX-expressing Ad vector (dE1-RGD/LacZ/ RLX) into scar tissue on the skin of a pig model. A previous investigation from our lab demonstrated that RLX-expressing Ad has potential therapeutic effects on keloid and hypertrophic scars due to the reversal of pathological fibrosis. ${ }^{18-20}$ However, a replication-incompetent Ad that expressed relaxin (AdRLX) vector system was limited by immunogenicity, local inflammation, a short half-life, enzymatic inactivation, and transient effects, which resulted in short-term expression of RLX. ${ }^{21}$ To address this problem, we tested a matrix-based depot system with a biodegradable alginate gel, which is a natural polymer frequently used in biomedical applications. Alginate gel can be used as a depot system for sustained release of Ad and maintenance of Ad viral activity due to its biocompatible environment. ${ }^{22,23}$ Thus, the use of alginate gel should ultimately increase therapeutic efficacy of RLX.

\section{MATERIALS AND METHODS}

\section{Scar formation}

The scar model was created with three Yorkshire pigs (XP Bio, Anseong, Korea; four months of age, $40 \mathrm{~kg}$ ). Anesthesia was induced in each pig via intramuscular injection of Zoletil (5 mg/ $\mathrm{kg}$, Virbac, Carros, France) and Rompun (2 mg/kg, Bayer, Seoul, Korea). Hair was then completely removed from the backs and bellies of each pig. Inhalation anesthesia was achieved with isoflurane (IsoFlo, Abbott Laboratories, Abbott Park, IL, USA). After anesthetizing the pigs, 36 full-thickness skin defects $(3 \times 3$ $\mathrm{cm}^{2}$ ) were created symmetrically on the back of each Yorkshire pig with 18 wounds on each side of the midline. Each wound reached the depth of the muscle fascia to mimic scar formation. The distance of each wound was maintained $5 \mathrm{~cm}$ in the horizontal direction and $3 \mathrm{~cm}$ in the vertical direction to control the interval of each scar (Supplementary Fig. 1, only online). Intravenous antibiotics and wound dressings with TegaDerm $^{\circledast}$ (3M, St. Paul, MN, USA) were administered postoperatively for 5 days, after which open dressings were maintained. Scar tissue, which was distinct in appearance from that of the peripheral normal tissue, formed at 50 days after creating the initial skin defect (Supplementary Fig. 2, only online). ${ }^{7}$ The Animal Care and Experiment Committee of Yonsei University approved the experimental protocol (2011-0181).

\section{Preparation of Ad and alginate gel}

A replication-incompetent Ad that expressed relaxin (Ad-RLX) and control Ad (Ad-LacZ) ${ }^{18}$ was used in this study. The propagation, purification, and titration of Ad were performed as previously described.$^{24,25}$ An alginate (alginic acid sodium salt, Sigma, St. Louis, MO, USA) solution of $5 \mathrm{wt} . \%$ was prepared with $0.5 \mathrm{~g}$ of alginate and $0.09 \mathrm{~g}$ of $\mathrm{NaCl}$ (Sigma) in $10 \mathrm{~mL}$ of phosphate-buffered saline. The alginate solution was stirred for $24 \mathrm{~h}$ at room temperature and gelated at various concentrations of $\mathrm{CaCl}_{2}$ (Sigma), ranging from 20 to $200 \mathrm{mM}$, to determine the optimal conditions for Ad transduction.

\section{Injection of Ad into the pig scar model}

Wound epithelialization and scar formation occurred on postoperative day 50 . Anesthesia was induced in each pig by intramuscular injection of Zoletil ( $5 \mathrm{mg} / \mathrm{kg}$; Virbac) and Rompun ( $2 \mathrm{mg} / \mathrm{kg}$; Bayer). Hair was then completely removed from the backs and bellies of each pig. Scars were divided into three groups (gel, gel/Ad-LacZ and gel/Ad-RLX). We injected $1 \mathrm{~mL}$ of alginate gel, the same amount of alginate gel encapsulating dEl-RGD/LacZ [ $5 \times 10^{7}$ plaque forming unit (PFU)], or alginate 
gel encapsulating dE1-RGD/LacZ/RLX in each group respectively. Injections were performed with a 27-gauge needle and 1-mL syringe, and directly into the intradermal layers of the scar regions.

\section{Therapeutic evaluation of scar area, color index, and pliability}

The surface areas of scars were imaged with a digital camera. The images of the surface areas of the scars were measured with a ruler and compared to a $1-\mathrm{cm}^{2}$ standard. Measurements were assessed with Image J software (National Institutes of Health, Bethesda, MD, USA). The color of the scar was quantitatively analyzed with a spectrophotometer (CM-700D; Konica Minolta, Inc., Tokyo, Japan). The color of each scar was examined with melanin (the degree of scar darkness) and erythema (the degree of scar redness) indices. ${ }^{26}$ Pliability was measured with a durometer (H1000 Mini-Dial, RexGauge Co., Buffalo Grove, IL, USA). Each value was measured more than three times, and the average value was calculated. All of the evaluations were performed every 10 days until the 50th day after virus injection by the same individual who was blinded to the experimental group. Scar area, color index, and pliability were measured by an independent blinded physician.

\section{Immunofluorescence microscopy}

Specimens were collected at 10 days after the administration of the Ad virus and were embedded in paraffin blocks. Paraffin-embedded tissues were sectioned, deparaffinized, hydrated, and treated with blocking buffer (20\% normal goat serum, S-100, Vector Lab Inc., Burlingame, CA, USA) for one hour. After removing the blocking buffer, sections were immersed in primary antibody (anti-relaxin, Santa Cruz Biotechnology, Dallas, TX, USA) overnight at $4^{\circ} \mathrm{C}$ and then incubated in secondary antibody (DyLight594, Vector Lab Inc.) at room temperature for 2 hours. The nucleus was stained with mounting solution containing DAPI. The degree of RLX expression in the tissue was evaluated by confocal microscopy (LSM700, Carl Zeiss, Oberkochen, Germany).

\section{Arrangement of collagen fibers and mast cell count}

Picrosirius red (Sigma-Aldrich Co., St. Louis, MO, USA) and toluidine blue (Sigma-Aldrich Co.) staining was performed on specimens 50 days after virus injection. Full thickness skin tissues, $1 \times 1 \mathrm{~cm}$, were obtained from the center areas of scars and embedded in paraffin: we obtained $5 \mu \mathrm{m}$ sections. Using the stained tissues, the number of mast cells (toluidine blue staining) and the collagen arrangement (picrosirius red staining) were compared using optical microscopy (BX51, Olympus, Tokyo, Japan). The number of mast cells was counted in five different areas randomly chosen for each slide, and counting was performed by magnifying images under $\times 100$ magnification.

\section{Immunohistochemistry}

Scar tissue sections were incubated at $4^{\circ} \mathrm{C}$ overnight with one of the following primary antibodies: mouse anti-collagen type-I (ab6308; Abcam, Ltd., Cambridge, UK), mouse anti-collagen type-III (C7805; Sigma-Aldrich Co.), mouse anti-elastin (E4013; Sigma-Aldrich Co.), mouse anti-fibronectin (sc-52331; Santa Cruz Biotechnology), rabbit anti-transforming growth factor- $\beta 1$ (anti-TGF- $\beta 1$ ) (ab9758; Abcam, Ltd.), or mouse antinatural killer-1.1 (NK-1.1) antibody (108701; Biolegend, San Diego, CA, USA). Tissues were then incubated at room temperature for 20 min with the DAKO Envision ${ }^{\mathrm{TM}}$ Kit (DAKO, Glostrup, Denmark) as a secondary antibody. Expression levels of type-I and type-III collagens, elastin, fibronectin, TGF- $\beta 1$, and NK-1.1 were semi-quantitatively analyzed with MetaMorph $^{\circledR}$ image analysis software (Universal Image Corp., Buckinghamshire, UK). Results are expressed as the mean optical density of six different digital images. Immunohistochemistry was performed by the same individual who was blinded to the experimental group, and the tissue sections for analysis were obtained from the center areas of scar tissue.

\section{Real-time reverse transcriptase-polymerase chain} reaction for expression of TGF- $\beta 1$ and TGF- $\beta 3$ mRNAs Scar tissues were treated with gel, gel/Ad-LacZ, or gel/Ad-RLX at $5 \times 10^{7} \mathrm{PFU}$ of Ad. On the injection date and 50 days posttreatment, cDNA was synthesized with AccuPower ${ }^{\mathrm{TM}}$ RT PreMix (Bioneer, Daejeon, Korea) after extracting total RNA with TRIzol reagent (Invitrogen, Carlsbad, CA, USA). PCR was performed in a 20- $\mu \mathrm{L}$ reaction volume containing the synthesized cDNA and $2 \times$ Taq Premix (Solgent, Daejeon, Korea). Thermal cycling was performed initially for $5 \mathrm{~min}$ at $94^{\circ} \mathrm{C}$, followed by 30 cycles of $30 \mathrm{sec}$ at $94^{\circ} \mathrm{C}, 30 \mathrm{sec}$ at $58^{\circ} \mathrm{C}$, and $30 \mathrm{sec}$ at $72^{\circ} \mathrm{C}$, and finally $5 \mathrm{~min}$ at $72^{\circ} \mathrm{C}$. After electrophoresis on a $1 \%$ agarose gel, each band was evaluated by densitometry (Image J, National Institutes of Health). The standard value ( $\beta$-actin) was converted to 1 , and analyses were performed by obtaining the ratio with the other measured values [TGF- $\beta 1$ (Ss03382325, ThermoFisher Scientific, MA, USA) and TGF- $\beta 3$ (Ss03394349, ThermoFisher Scientific)]. PCR was performed three times for each mRNA in each tissue, and the average value was calculated.

\section{Statistical analysis}

All test results are presented as a mean \pm standard error of the mean. The results of quantitative analyses were statistically analyzed in three groups by one-way ANOVA and the multiple-comparison Bonferroni correction method. A $p$ value less than 0.05 was considered statistically significant. Statistical analyses were performed with SAS software (version 9.1.3, SAS Institute Inc., Cary, NC, USA). 


\section{RESULTS}

\section{Relaxin-expressing Ad decreases scar size, color index, and pliability in pig scar tissue}

Scars with full thickness were generated on the backs of Yorkshire pigs. An RLX-expressing Ad vector entrapped in alginate gel was evaluated for effects on scar remodeling. The sizes of the initial scars were $3.15 \pm 0.15 \mathrm{~cm}^{2}, 3.12 \pm 0.11 \mathrm{~cm}^{2}$, and $3.18 \pm$ $0.08 \mathrm{~cm}^{2}$ in the gel, gel/Ad-LacZ, and gel/Ad-RLX groups, respectively. Alginate gel (gel), alginate gel encapsulating dE1RGD/LacZ (gel/Ad-LacZ), or alginate gel encapsulating dE1RGD/LacZ/RLX (gel/Ad-RLX), respectively, was injected into the scar tissue. The sizes of the scars decreased to $1.61 \pm 0.15$ $\mathrm{cm}^{2}, 1.70 \pm 0.10 \mathrm{~cm}^{2}$, and $1.37 \pm 0.05 \mathrm{~cm}^{2}$ in each group, respectively, by 50 days after treatment (Fig. 1A and B, Supplementary Fig. 3, only online). These results indicated that RLX expression from Ad (gel/Ad-RLX) reduces the size of scars compared to those of the control groups (gel or gel/Ad-LacZ) $(p<0.05$; Fig. 1B).

To further examine the effect of RLX on scar remodeling, color changes and pliability were investigated by spectrophotometry in conjunction with erythema and melanin indices and a durometer. The erythema index values of the initial scars were $1.97 \pm 0.20,2.01 \pm 0.10$, and $2.01 \pm 0.09$ in the gel, gel/AdLacZ, and gel/Ad-RLX groups, respectively. Erythema was significantly reduced $(1.52 \pm 0.15 ; p<0.05)$ in the gel/Ad-RLX group at 50 days after treatment, compared to those in the gel $(2.07 \pm 0.35)$ and gel/Ad-LacZ (2.05 \pm 0.08$)$ groups (Fig. 1C). The initial melanin index values were $0.23 \pm 0.06,0.23 \pm 0.02$, and
$0.23 \pm 0.03$ in the gel, gel/Ad-LacZ, and gel/Ad-RLX groups, respectively (Fig. 1D). This index was also significantly $(p<0.05)$ decreased to $0.08 \pm 0.02$ in the gel/Ad-RLX group, compared to $0.21 \pm 0.05$ and $0.21 \pm 0.04$ in the gel and gel/Ad-LacZ control groups, respectively, at 50 days after treatment. Similarly, pliability was significantly $(p<0.05)$ reduced in the gel/Ad-RLX group, compared to the gel and gel/Ad-LacZ control groups (Fig. 1E). The initial scars had pliability measurements of $20.50 \pm 1.42$, $19.73 \pm 0.88$, and $19.08 \pm 1.06$ in the gel, gel/Ad-LacZ, and gel/ Ad-RLX groups, respectively. Pliability was reduced to $18.00 \pm$ $0.58,17.46 \pm 1.62$, and $11.15 \pm 1.72$ in the gel, gel/Ad-LacZ, and gel/Ad-RLX groups, respectively, by 50 days after treatment. Taken together, these data suggested that RLX expression from gel/Ad-RLX promotes the remodeling of scar tissue.

\section{Relaxin-expressing Ad induces collagen rearrangement and decreases expression of major ECM components in scar tissue}

We examined RLX expression by immunofluorescence staining in pig scar tissues transduced with gel, gel/Ad-LacZ, or gel/Ad-RLX. The gel/Ad-RLX-treated groups showed markedly increased immunoreactivity for RLX, compared to the control groups (gel or gel/Ad-LacZ). These results confirmed an association between increased RLX expression and reduced scar tissue formation in tissues transduced with the gel/AdRLX depot system (Fig. 2).

To evaluate the effects of RLX-expressing Ad on collagen-fiber arrangement, tissues were stained with picrosirius red, which binds specifically to collagen fibrils of various diame-
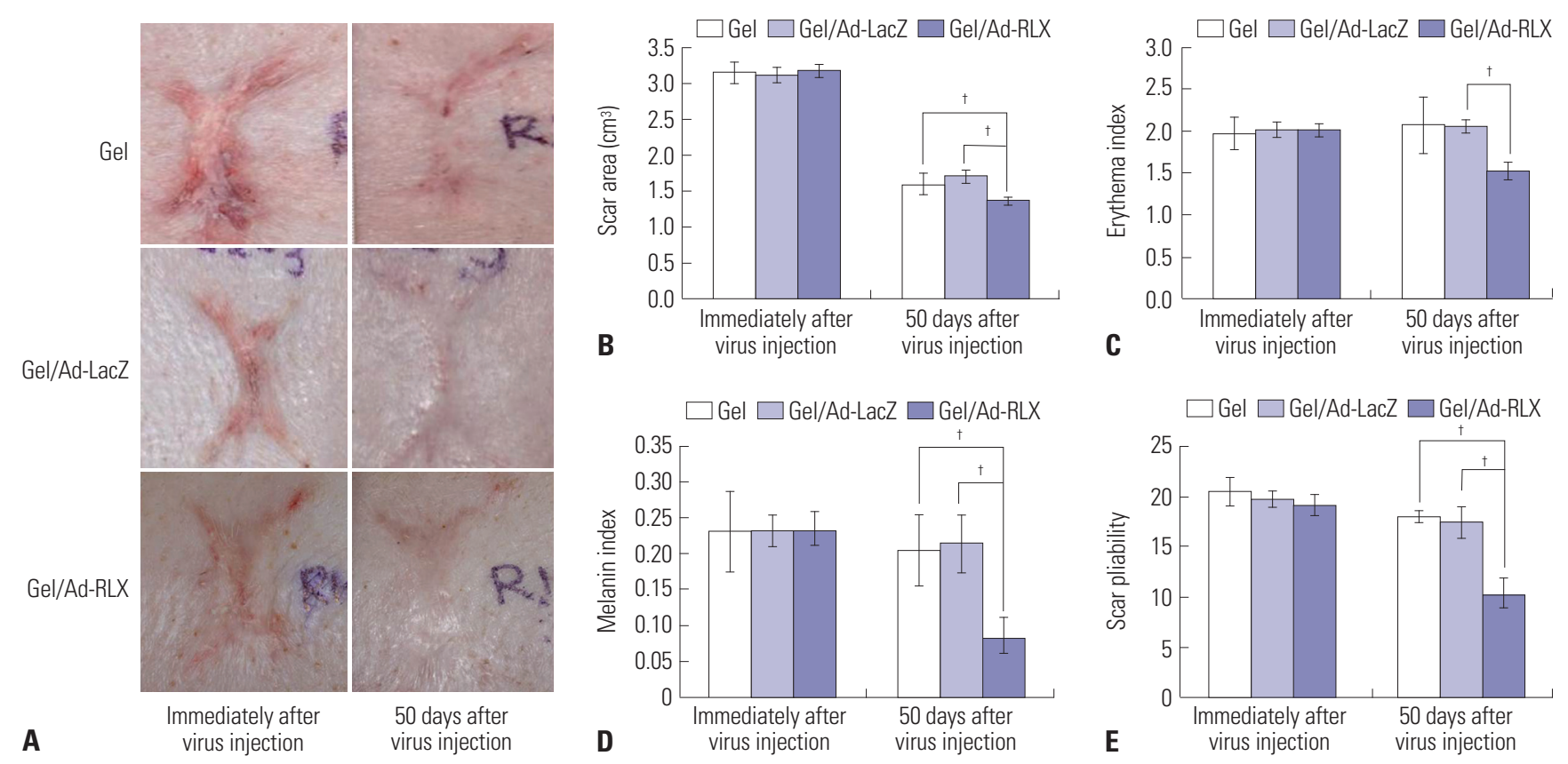

Fig. 1. Relaxin (RLX) expression reduces the size, color index, and pliability of pig scars. (A) Scars were created on the backs of pigs. The area of scars decreased, and the color improved at 50 days after the injection of relaxin-expressing Ad loaded in alginate gel (gel/Ad-RLX). (B) Scar size, (C) erythema index values, (D) melanin index values, and (E) pliability were significantly reduced $\left({ }^{\dagger} p<0.05\right)$ compared to those of control groups [gel or LacZ-expressing Ad loaded in alginate gel (gel/Ad-LacZ)]. Data are expressed as a mean \pm standard error of the mean. 


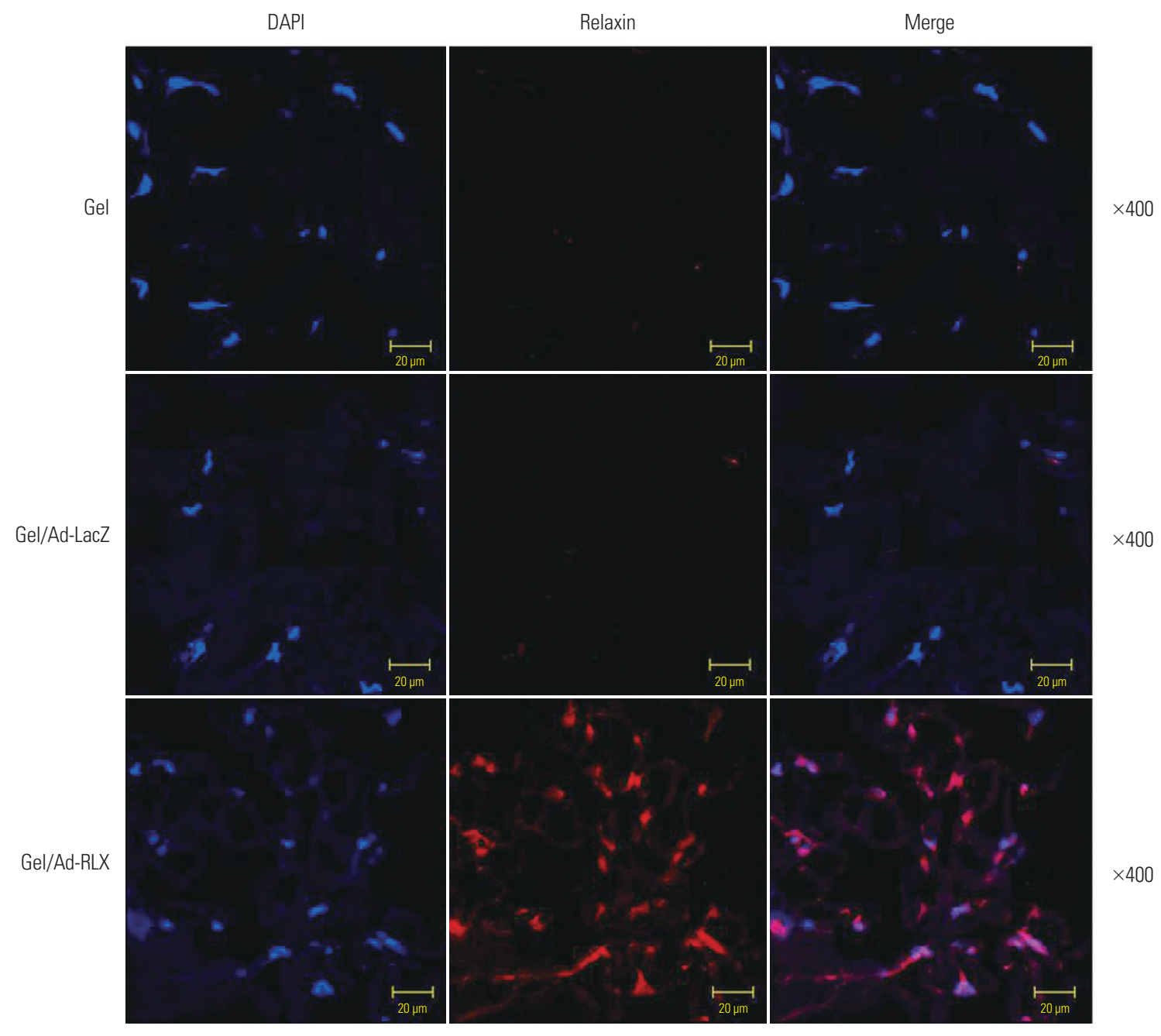

Fig. 2. Immunofluorescence staining to evaluate the effects of relaxin on scar reduction in pig scar tissues. Increased expression of relaxin was detected after treatment with relaxin-expressing Ad loaded in alginate gel (gel/Ad-RLX). In contrast, control groups [gel or LacZ-expressing Ad loaded in alginate gel (gel/Ad-LacZ)] showed lower levels of relaxin. Nuclei were visualized by DAPI (4',6-diamidino-2-phenylindole) staining; original magnification, $\times 400$; scale bar $=20 \mu \mathrm{m}$.

ters. The gel/Ad-RLX-treated groups had closely packed collagen fibers and formed bundles of collagen (Fig. 3A), compared to those in the control groups (gel or gel/Ad-LacZ). These data suggested that RLX induces collagen rearrangement to resemble that of mature, bundle-shaped collagen fibers.

We next examined the effects of RLX overexpression on the major extracellular matrix (ECM) components of scar tissues. Immunohistochemical staining of scar sections revealed significant reductions in type-I collagen, type-III collagen, elastin, and fibronectin in the gel/Ad-RLX-treated group, compared with those in the gel/Ad-LacZ group $(p<0.01)$ (Fig. 3B-F). These data strongly suggested that expression levels of the major ECM components were significantly decreased by RLX overexpression in pig scar tissues. Moreover, these results implied that RLX can play a prominent role in ECM remodeling during the development of scar tissue.
Relaxin-expressing Ad increases MMP-1 and decreases TIMP-1 and $\alpha$-SMA expression in pig scar tissue Matrix metalloproteinase-1 (MMP-1), tissue inhibitor of metalloproteinase-1 (TIMP-1), and alpha-smooth muscle actin ( $\alpha$-SMA) are important markers of the effects of TGF- $\beta$ on wound repair. Therefore, we performed immunohistochemistry to examine expression of MMP-1, TIMP-1, and $\alpha$-SMA. MMP-1 expression levels were significantly increased by 7.0 -fold in pig scar tissues treated with gel/Ad-RLX versus that of the gel/AdLacZ-treated tissues ( $p<0.01)$ (Fig. 4). In contrast, TIMP-1 and $\alpha$-SMA expression levels were significantly decreased by 1.9 and 9.9-fold, respectively, in pig scar tissues treated with gel/ Ad-RLX transduction $(p<0.05)$. These results suggested that RLX upregulates MMP-1 and downregulates TIMP-1 and a-SMA, which are major players in collagen breakdown. 

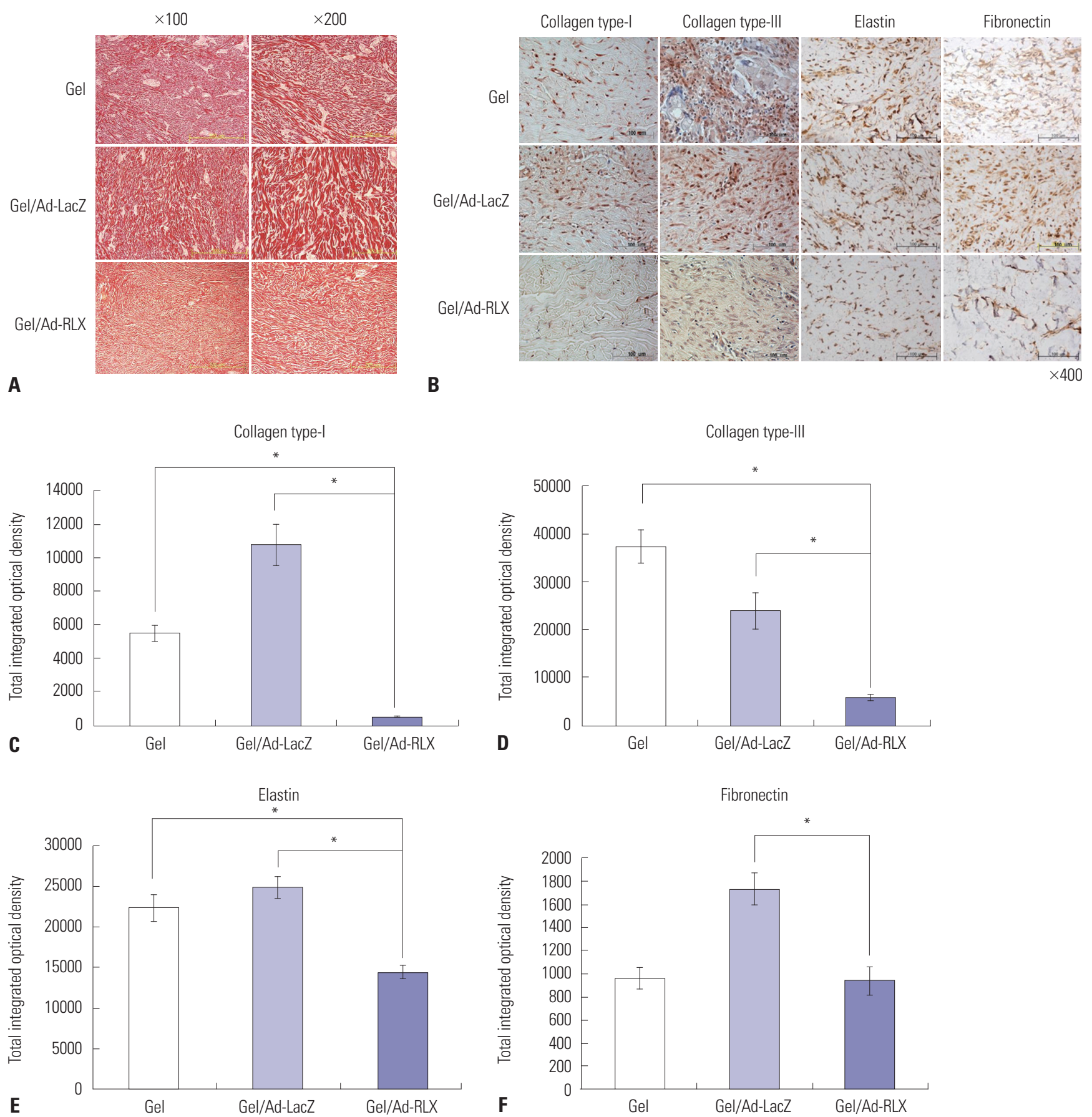

Fig. 3. Picrosirius red staining and immunohistochemical staining for collagen type-I, collagen type-III, elastin, and fibronectin in pig scar tissues. (A) Dense and coarse collagen fibers were replaced with closely packed collagen fibers after pig scar tissues were treated with gel/Ad-RLX; original magnification, $\times 100$ and $\times 200$; scale bar $=500 \mu \mathrm{m}$ and $200 \mu \mathrm{m}$. (B) The expression levels of the major extracellular matrix components collagen type-I, collagen type-III, elastin, and fibronectin were lower in pig scar tissues treated with gel/Ad-RLX than those in pig tissues treated with gel or gel/AdLacZ; original magnification, $\times 400$; scale bar=100 $\mu \mathrm{m}$. Semi-quantitative image analyses revealed that (C) collagen type-I, (D) collagen type-III, (E) elastin, and (F) fibronectin were significantly decreased in pig tissues treated with gel/Ad-RLX, compared to those in pig tissues treated with control virus $\left(^{*} p<0.01\right)$. Data are expressed as a mean \pm standard error of the mean.

\section{Relaxin-expressing Ad downregulates TGF- $\beta 1$ and upregulates TGF- $\beta 3$ expression in pig scar tissue}

To examine the mechanism by which RLX-expressing Ad suppresses expression of the major ECM components, TGF- $\beta 1$ and TGF- $\beta 3$ mRNA levels were examined in scar tissues. The
gel/Ad-RLX group showed decreased TGF- $\beta 1$ mRNA expression and increased TGF- $\beta 3$ mRNA expression in scar tissues, compared with those in the gel or gel/Ad-LacZ group (Fig. 5) $(p<0.05)$, suggesting that the reduced expression of ECM components by RLX overexpression is associated with decreased 


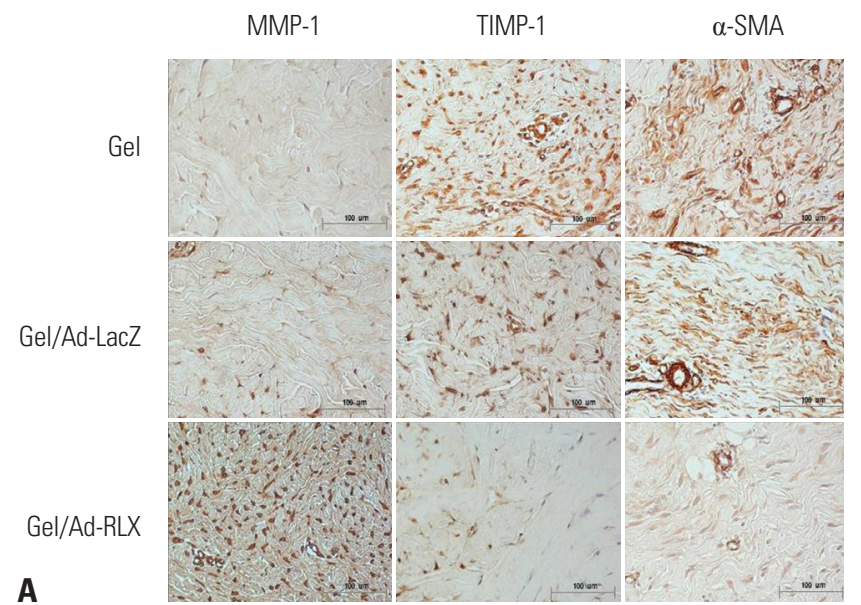

TIMP-1

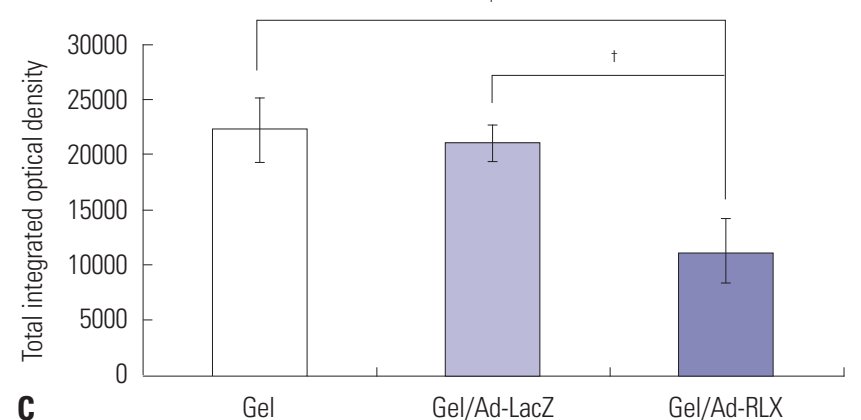

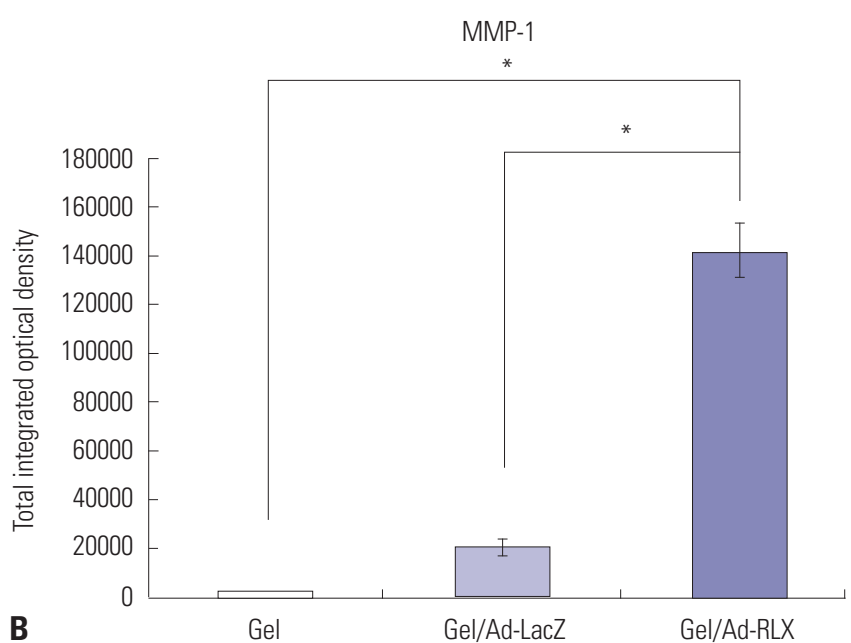

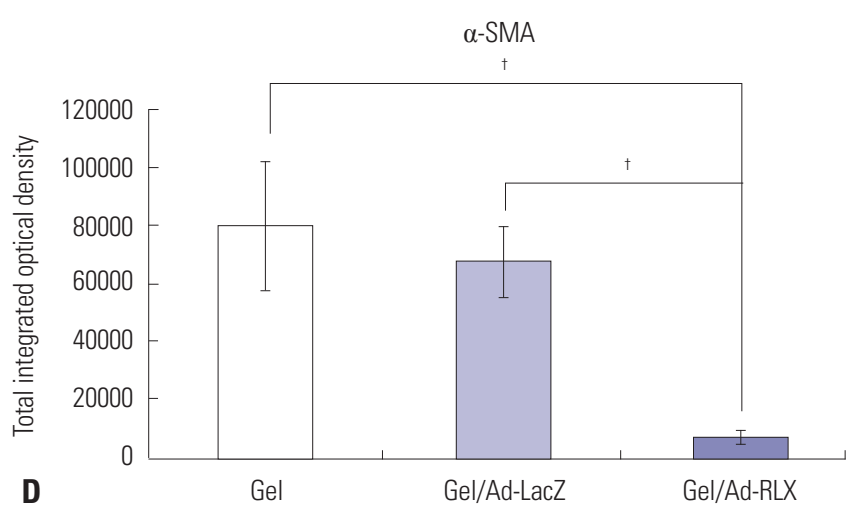

Fig. 4. Immunohistochemical staining for matrix metalloproteinase-1 (MMP-1), tissue inhibitor of metalloproteinase-1 (TIMP-1), and alpha-smooth muscle actin ( $\alpha$-SMA) in pig scar tissues. (A) Treatment with gel/Ad-RLX markedly increased MMP-1 levels and decreased TIMP-1 and $\alpha-S M A$ levels in pig scar tissues; original magnification, $\times 400$; scale bar $=100 \mu \mathrm{m}$. Semi-quantitative image analyses revealed that (B) MMP-1 was significantly increased in pig tissues treated with gel/Ad-RLX versus that of tissues treated with control virus (* $p<0.01)$. (C) TIMP-1 and (D) $\alpha$-SMA levels were significantly decreased in pig tissues treated with gel/Ad-RLX, compared to those with control virus $\left({ }^{\dagger} p<0.05\right)$. Data are expressed as mean \pm standard error of the mean.

expression of TGF- $\beta 1$ and increased expression of TGF- $\beta 3$.

We also checked TGF- $\beta 1$ expression via immunohistochemical staining of the scar sections. The results revealed significant reductions in TGF- $\beta 1$ in the gel/Ad-RLX-treated group, compared to the gel/Ad-LacZ group $(p<0.05)$ (Supplementary Fig. 4, only online).

\section{Relaxin-expressing Ad decreases mast cell counts and NK-1.1 expression in pig scar tissue}

Mast cells are reported to be involved in the proliferation and contraction of fibroblasts, and the synthesis of ECM. They also play a key role in scar formation. The amount of mast cells was increased by four times in hypertrophic scars than in normal skin. ${ }^{27-29}$ To examine the local inflammatory effects of RLX-expressing Ad, mast cell counts and NK-1.1 immunohistochemistry were assessed. Immediately after scar formation, the mean numbers of mast cells were $6.20 \pm 1.30,7.00 \pm 0.80$, and $6.30 \pm$ 0.70 in the gel, gel/Ad-LacZ, and gel/Ad-RLX groups, respectively, with no significant differences. Fifty days after injection of the virus, the mean numbers of observed mast cells were $6.80 \pm$
0.42 in the gel group and $7.09 \pm 0.68$ in the gel/Ad-LacZ group. A significant decrease was seen in the mean number of mast cells in the gel/Ad-RLX group ( $4.89 \pm 0.40 ; p<0.01$; Supplementary Fig. 5, only online). NK-1.1 expression was significantly lower in the gel/Ad-LacZ and gel/Ad-RLX-treated groups, compared to the gel group $(p<0.05)$ (Supplementary Fig. 6, only online).

\section{DISCUSSION}

Scars, which are caused by collagen deposition, confer a large number of functional problems. Nowadays, many patients want to minimize scar formation, and many treatment modalities, such as scar revision, laser therapy, and medications, have been applied to prevent scar formation. However, the successful treatment of scars has not been fully resolved. ${ }^{30-32}$

This study was performed by adopting a pig scar model used our previous study. The aim of this study was to verify the antifibrotic effect of RLX on scars. Recent studies supported our 

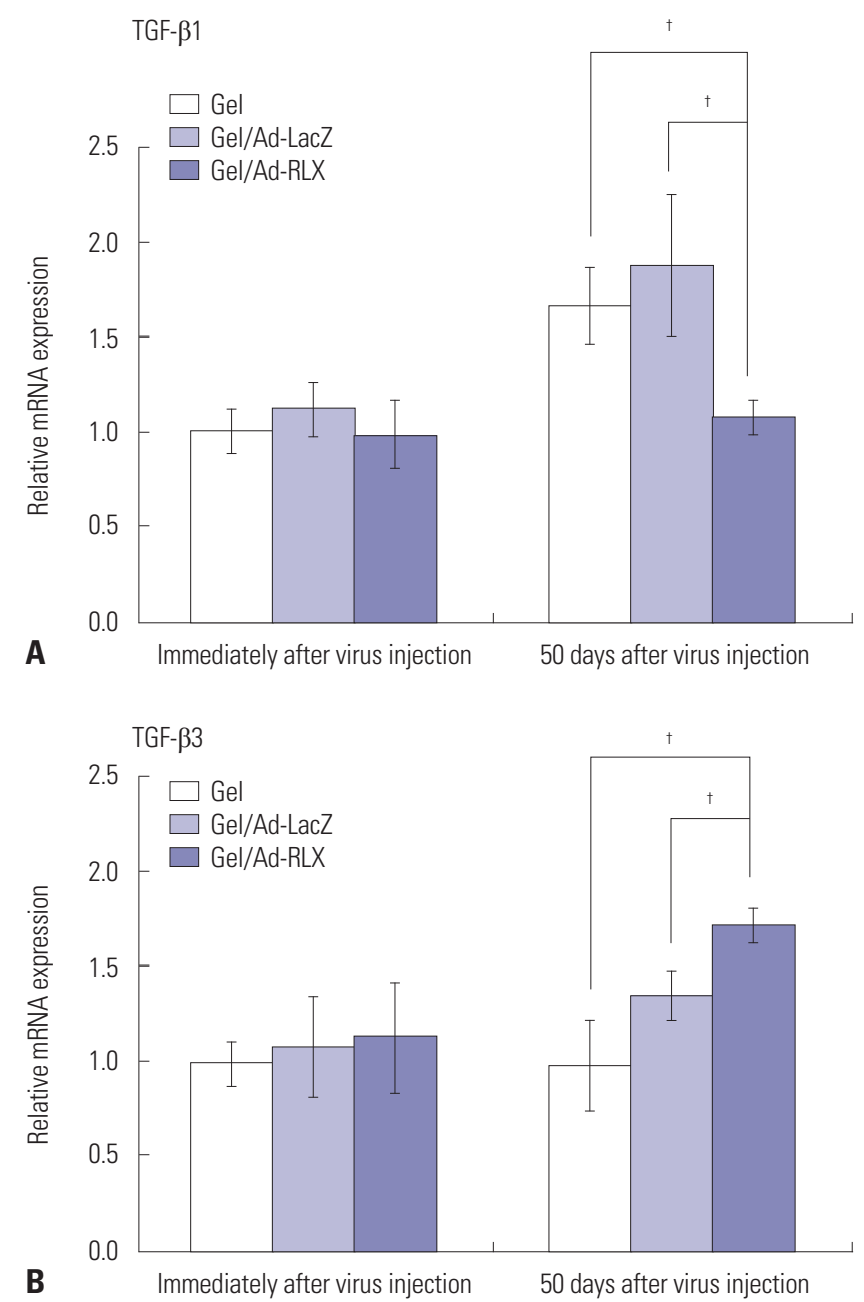

Fig. 5. Relaxin-expressing Ad decreases transforming growth factor- $\beta 1$ (TGF- $\beta 1$ ) and increases TGF- $\beta 3$ mRNA levels in pig scar tissues. (A) Quantitative analyses by qRT-PCR indicated that TGF- $\beta 1$ mRNA levels were significantly decreased $\left({ }^{\dagger} p<0.05\right)$ in scar tissues treated with gel/ $\mathrm{Ad}-\mathrm{RLX}$, compared with those of tissue treated with control virus. (B) In contrast, TGF- $\beta 3$ mRNA was significantly increased $\left({ }^{\dagger} p<0.05\right)$ by relaxin overexpression in pig scar tissues. Data are expressed as a mean \pm standard error of the mean.

data and suggested that RLX exerts alternative biological effects mediated directly through TGF- $\beta$-dependent signaling. ${ }^{14,18,19,30-32}$ These effects elicit downregulation of TGF- $\beta 1$ and upregulation of TGF- $\beta 3$ to inhibit myofibroblast accumulation, collagen synthesis/secretion, and ultimately, fibrosis progression in scar tissue. Other studies have suggested that TGF- $\beta 1$ is a potent fibrogenic growth factor, whereas TGF- $\beta 3$ reduces scar tissue by decreasing synthesis and increasing degradation of type-I collagen..$^{33}$ TGF- $\beta$ plays a key role in the pathophysiology of fibroplasia by decreasing the expression of MMPs and increasing the expression of TIMPs. ${ }^{19}$

Furthermore, TGF- $\beta$ induces $\alpha$-SMA expression, implying that $\alpha$-SMA expression is a marker of TGF- $\beta$ activity. ${ }^{32-37}$ Thus, the increased expression of MMP-1 and decreased levels of TIMP and $\alpha$-SMA suggest that dE1-RGD/LacZ/RLX plays a prominent role in ECM remodeling in hypertrophic scars. MMP-1 promotes degradation and ECM remodeling, whereas TIMP- 1 decreases the activities of plasminogen activators, such as MMPs, and shifts the ECM equilibrium toward degradation. Thus, increased MMP-1 production and decreased TIMP-1 expression should reduce the amount of abnormal or unfolded collagen during wound healing. In addition, reduced levels of $\alpha$-SMA may be a positive indicator of TGF- $\beta$ activity, which was decreased in the gel/Ad-RLX group in this study. Taken together, these studies suggest that dE1-RGD/LacZ/ RLX is a potential therapeutic intervention for hypertrophic scars.

Ad has been used extensively as a gene-delivery system in experimental models of cancer and cardiovascular diseases. ${ }^{38-40}$ However, one of the chief concerns with translating these experimental results to the clinical setting is the large and repeated dose that is usually required to generate a modest clinical effect or to achieve the desired therapeutic concentration at the target. To address this concern, we utilized an Arg-GlyAsp-modified (RGD) replication-incompetent Ad vector as a strategy to allow direct delivery of the RLX gene to wounds. Previous studies from our lab showed that RGD-modified Ad markedly increases gene transfer efficiency in primary keloid fibroblasts. Thus, we believe that RGD-modified Ad is a highly efficient gene-transfer vehicle that does not adversely affect the replication potential of target cells. ${ }^{14,17}$

To further advance this potential therapy for hypertrophic scars, we generated and characterized an alginate gel-matrix system to entrap RLX-expressing Ad as a delivery vehicle..22,23 Previous studies have indicated that the biological activity of Ad loaded in alginate gel is prolonged, compared with that of naked Ad, over an extended period of time. Cells that were transduced with gel-released Ad expressed green fluorescence protein at a 13-fold greater intensity at seven days post-incubation compared to that of naked Ad. Because long-term transduction is needed for in vivo applications, the $\mathrm{Ad} /$ alginate gel may be very useful as a depot system. The Ad/alginate gel acts as a reservoir that releases Ad in a sustained manner and maintains the biological activity of Ad. Alginate gel has also been shown to limit the mobility of Ad by entrapping Ad within its scaffold, further minimizing the spread of the replication-incompetent Ad outside of the scar tissue. These results show that sustained and controlled delivery of Ad in alginate gel markedly augments therapeutic effects, prolongs maintenance of Ad activity, and provides specificity for local delivery of replication-incompetent Ad at high concentrations. These advantages of the $\mathrm{Ad} /$ alginate system will allow for potent local viral therapy to a target site.

In conclusion, we provide strong evidence that RLX promotes the remodeling of scar tissue. Moreover, sustained and controlled delivery of Ad in alginate gel markedly augments the therapeutic effects of RLX. Our data support further evaluation of gel/Ad-RLX as a novel gene-therapy system for treat- 
ing human scars.

\section{ACKNOWLEDGEMENTS}

This work was supported by a faculty research grant of Yonsei University College of Medicine (6-2012-0037, Dr. In Sik Yun), a grant from Hanyang University of Korea (HY-2011-G-2011 00000001880; Dr. Chae-Ok Yun) and the National Research Foundation of Korea (NRF-2018R1D1A1B07042537, Dr. In Sik Yun; 2016M3A9B5942352, Dr. Chae-Ok Yun). Hyo Min Ahn is a graduate student sponsored by the Brain Korea 21 plus program, Hanyang University, Seoul, South Korea (22A2013001 1095).

\section{AUTHOR CONTRIBUTIONS}

Conceptualization: Won Jai Lee and Chae-Ok Yun. Data curation: Eunhye Kang. Formal analysis: Hyo Min Ahn. Funding acquisition: Chae-Ok Yun. Investigation: In Sik Yun. Project administration: Yong Oock Kim. Supervision: Dong Kyun Rah and Chae-Ok Yun. Validation: Won Jai Lee. Writing_original draft: In Sik Yun. Writing_review \& editing: Tai Suk Roh.

\section{ORCID iDs}

$\begin{array}{ll}\text { In Sik Yun } & \text { https://orcid.org/0000-0003-1103-7047 } \\ \text { Eunhye Kang } & \text { https://orcid.org/0000-0002-8094-0726 } \\ \text { Hyo Min Ahn } & \text { https://orcid.org/0000-0002-1268-8637 } \\ \text { Yong Oock Kim } & \text { https://orcid.org/0000-0002-3756-4809 } \\ \text { Tai Suk Roh } & \text { https://orcid.org/0000-0001-8681-159X } \\ \text { Won Jai Lee } & \text { https://orcid.org/0000-0003-3056-0503 } \\ \text { Chae-Ok Yun } & \text { https://orcid.org/0000-0002-9466-4531 }\end{array}$

\section{REFERENCES}

1. Desmoulière A, Darby IA, Gabbiani G. Normal and pathologic soft tissue remodeling: role of the myofibroblast, with special emphasis on liver and kidney fibrosis. Lab Invest 2003;83:1689-707.

2. Le AD, Brown JJ. Wound healing: repair biology and wound and scar treatment. In: Bagheri SC, Bell RB, Khan HA, editors. Current therapy in oral and maxillofacial surgery. 1st ed. Philadelphia: Saunders; 2012. p.6-10.

3. Bae SH, Bae YC. Analysis of frequency of use of different scar assessment scales based on the scar condition and treatment method. Arch Plast Surg 2014;41:111-5.

4. Corr DT, Gallant-Behm CL, Shrive NG, Hart DA. Biomechanical behavior of scar tissue and uninjured skin in a porcine model. Wound Repair Regen 2009;17:250-9.

5. Wang XQ, Kravchuk O, Liu PY, Kempf M, Boogaard CV, Lau P, et al. The evaluation of a clinical scar scale for porcine burn scars. Burns 2009;35:538-46.

6. Wang XQ, Liu PY, Kempf M, Cuttle L, Chang AH, Wong M, et al. Burn healing is dependent on burn site: a quantitative analysis from a porcine burn model. Burns 2009;35:264-9.

7. Yun IS, Jeon YR, Lee WJ, Lee JW, Rah DK, Tark KC, et al. Effect of human adipose derived stem cells on scar formation and remodeling in a pig model: a pilot study. Dermatol Surg 2012;38:1678-88.

8. Zhao S, Lee HY, Sherwood OD. Porcine and human relaxin bioactivity: bioactivities of porcine relaxin and human relaxin do not differ in mice and rats. Ann N Y Acad Sci 2005;1041:126-31.

9. Samuel CS, Hewitson TD, Unemori EN, Tang ML. Drugs of the future: the hormone relaxin. Cell Mol Life Sci 2007;64:1539-57.

10. Sherwood OD. Relaxin's physiological roles and other diverse actions. Endocr Rev 2004;25:205-34.

11. Samuel CS, Hewitson TD. Relaxin in cardiovascular and renal disease. Kidney Int 2006;69:1498-502.

12. Dschietzig T, Bartsch C, Baumann G, Stangl K. Relaxin-a pleiotropic hormone and its emerging role for experimental and clinical therapeutics. Pharmacol Ther 2006;112:38-56.

13. Unemori EN, Erikson ME, Rocco SE, Sutherland KM, Parsell DA Mak J, et al. Relaxin stimulates expression of vascular endothelial growth factor in normal human endometrial cells in vitro and is associated with menometrorrhagia in women. Hum Reprod 1999;14: 800-6.

14. Kim JH, Lee YS, Kim H, Huang JH, Yoon AR, Yun CO. Relaxin expression from tumor-targeting adenoviruses and its intratumoral spread, apoptosis induction, and efficacy. J Natl Cancer Inst 2006; 98:1482-93.

15. Unemori EN, Lewis M, Constant J, Arnold G, Grove BH, Normand J, et al. Relaxin induces vascular endothelial growth factor expression and angiogenesis selectively at wound sites. Wound Repair Regen 2000;8:361-70.

16. Chartier C, Degryse E, Gantzer M, Dieterle A, Pavirani A, Mehtali M. Efficient generation of recombinant adenovirus vectors by homologous recombination in Escherichia coli. J Virol 1996;70: 4805-10.

17. Kim J, Cho JY, Kim JH, Jung KC, Yun CO. Evaluation of E1B geneattenuated replicating adenoviruses for cancer gene therapy. Cancer Gene Ther 2002;9:725-36.

18. Lee WJ, Choi IK, Lee JH, Lee JS, Kim YO, Rah DK, et al. Relaxinexpressing adenovirus decreases collagen synthesis and up-regulates matrix metalloproteinase expression in keloid fibroblasts: in vitro experiments. Plast Reconstr Surg 2012;130:407e-17e.

19. Lee WJ, Kim YO, Choi IK, Rah DK, Yun CO. Adenovirus-relaxin gene therapy for keloids: implication for reversing pathological fibrosis. Br J Dermatol 2011;165:673-7.

20. Lee WJ, Yun CO, Yun IS, Kim YO, Choi IK, Yun TJ, et al. Augmentation of rat skin flap viability by relaxin-expressing adenovirus. Wound Repair Regen 2011;19:709-17.

21. Ahi YS, Bangari DS, Mittal SK. Adenoviral vector immunity: its implications and circumvention strategies. Curr Gene Ther 2011;11: 307-20.

22. Choi JW, Kang E, Kwon OJ, Yun TJ, Park HK, Kim PH, et al. Local sustained delivery of oncolytic adenovirus with injectable alginate gel for cancer virotherapy. Gene Ther 2013;20:880-92.

23. Park H, Kim PH, Hwang T, Kwon OJ, Park TJ, Choi SW, et al. Fabrication of cross-linked alginate beads using electrospraying for adenovirus delivery. Int J Pharm 2012;427:417-25.

24. Choi KJ, Zhang SN, Choi IK, Kim JS, Yun CO. Strengthening of antitumor immune memory and prevention of thymic atrophy mediated by adenovirus expressing IL-12 and GM-CSF. Gene Ther 2012;19:711-23.

25. Zhang SN, Choi IK, Huang JH, Yoo JY, Choi KJ, Yun CO. Optimizing DC vaccination by combination with oncolytic adenovirus coexpressing IL-12 and GM-CSF. Mol Ther 2011;19:1558-68.

26. Yun IS, Lee WJ, Rah DK, Kim YO, Park BY. Skin color analysis using a spectrophotometer in Asians. Skin Res Technol 2010;16:311-5.

27. Kitamura Y, Oboki K, Ito A. Molecular mechanisms of mast cell development. Immunol Allergy Clin North Am 2006;26:387-405.

28. Sur R, Cavender D, Malaviya R. Different approaches to study mast cell functions. Int Immunopharmacol 2007;7:555-67.

29. Gilfillan AM, Rivera J. The tyrosine kinase network regulating mast 
cell activation. Immunol Rev 2009;228:149-69.

30. Park BY, Shin IS, Yun IS. Dovetail scar revision. Dermatol Surg 2012;38:1716-21.

31. Kim SG, Kim EY, Kim YJ, Lee SI. The efficacy and safety of ablative fractional resurfacing using a 2,940-Nm Er:YAG laser for traumatic scars in the early posttraumatic period. Arch Plast Surg 2012;39: 232-7.

32. Greenhalgh DG. Consequences of excessive scar formation: dealing with the problem and aiming for the future. Wound Repair Regen 2007; 15 Suppl 1:S2-5.

33. Shah M, Foreman DM, Ferguson MW. Neutralisation of TGF-beta 1 and TGF-beta 2 or exogenous addition of TGF-beta 3 to cutaneous rat wounds reduces scarring. J Cell Sci 1995;108(Pt 3):9851002.

34. Wilgus TA, Vodovotz Y, Vittadini E, Clubbs EA, Oberyszyn TM. Reduction of scar formation in full-thickness wounds with topical celecoxib treatment. Wound Repair Regen 2003;11:25-34.

35. Liu W, Chua C, Wu X, Wang D, Ying D, Cui L, et al. Inhibiting scar formation in rat wounds by adenovirus-mediated overexpression of truncated TGF-beta receptor II. Plast Reconstr Surg 2005;115: 860-70.

36. Gallant CL, Olson ME, Hart DA. Molecular, histologic, and gross phenotype of skin wound healing in red Duroc pigs reveals an abnormal healing phenotype of hypercontracted, hyperpigmented scarring. Wound Repair Regen 2004;12:305-19.

37. Margulis A, Nocka KH, Wood NL, Wolf SF, Goldman SJ, Kasaian MT. MMP dependence of fibroblast contraction and collagen production induced by human mast cell activation in a three-dimensional collagen lattice. Am J Physiol Lung Cell Mol Physiol 2009; 296:L236-47.

38. Samuel CS, Cendrawan S, Gao XM, Ming Z, Zhao C, Kiriazis H, et al. Relaxin remodels fibrotic healing following myocardial infarction. Lab Invest 2011;91:675-90.

39. Stewart DR. Scar prevention and cosmetically enhanced wound healing using relaxin. Ann N Y Acad Sci 2009;1160:336-41.

40. Du XJ, Xu Q, Lekgabe E, Gao XM, Kiriazis H, Moore XL, et al. Reversal of cardiac fibrosis and related dysfunction by relaxin. Ann N Y Acad Sci 2009;1160:278-84. 